\title{
Passive Acoustic Method in Bubble Size Distribution Determination
}

\author{
Stepan Gavrilev* and Mikhail Ivanov \\ B auman M oscow State Technical U niversity, Department of Ecology and Industrial Safety, M oscow, \\ Russia
}

\begin{abstract}
The paper is considering existing methods for determining the gas phase hydrodynamic properties in liquid medium. This paper presents a passive hydroacoustic method for determining the air bubbles size distribution in water. A dvantage of this method contrasting to the active ones lies in its invasiveness. M athematical model proposed for converting the spectrum of noise emitted by a cloud of bubbles into size distribution was tested in a number of experiments. Experiments were carried out in a glass cubic reservoir filled with water. Experiment results were verified by comparison with the photometric method.
\end{abstract}

\section{Introduction}

Development of technical systems in the energy industry, where two-phase gas-liquid flows are used, requires accurate monitoring the parameters of processes appearing in such systems. Gas bubbles size distribution in liquid medium is one of the key parameters, which attracted special attention in many works [1-3]. Optical, photometric and acoustic methods are used to solve such problems.

Optical and photometric methods are the mostly common employed [4-9], but they are having one significant drawback; these methods are applicable only in the optically transparent media; i.e. they could not be used in measuring the air bubbles size distribution in turbid liquid media. Therefore, acoustic methods are considered to be more versatile.

Most modern acoustic methods used in determining the bubbles size distribution are based on measuring attenuation and velocity of the acoustic wave passing through the bubble cloud layer. These methods are called active and are perfectly described in works [10-12]. But this approach has one minor drawback. Acoustic wave generated to irradiate the bubble cloud could affect the bubbles. This phenomenon may be seen in the results of work [13]. Authors of this work conducted a number of experiments accompanied by changing the emitted acoustic wave intensity, and each of the experiments obtained different results. Thus, in a number of cases, where it is especially important to maintain modes with a certain bubble size, using the active methods is unacceptable. Therefore, introduction of passive methods that do not affect dynamics of the processes under study is more preferable.

Passive methods are based on the fact that air bubbles in water are sources of acoustic signals. They emit an acoustic signal due to variable gas pressure inside a bubble.

* Corresponding author: stepan.tab92@gmail.com 
In 1933, Minnaert showed that under adiabatic conditions (heat exchange between bubble gas and liquid is negligible), the $f$ frequency of acoustic signal emitted by a gas bubble depends on its size in the following way:

$$
f=\frac{1}{2 \pi R} \sqrt{\frac{3 \gamma P}{\rho}}
$$

where: $f$ is the sound wave frequency emitted by the bubble; $P$ is the fluid absolute pressure; $\gamma$ is the gas specific heat capacity, $\rho$ is the fluid density; $R$ is the bubble radius.

This expression is valid for spherical bubbles. Bubble shape usually changes during the emergence. Depending on the size, they could be in the form of sphere, oblate spheroid, spherical segment or a mushroom cap. Work [14] presents criterion, according to which bubbles could be considered approximately spherical, if the following condition is satisfied:

$$
R<\delta_{\sigma},
$$

where: $R$ is the bubble radius; $\delta$ is the fluid capillary constant.

According to this criterion, bubbles in water are having the shape of a sphere, if their radius is $R<2.7 \mathrm{~mm}$.

The most complete description of bubble acoustics was given by Leighton (1994). According to work [15], the bubble emitted spectrum could be used to determine its size. Then, this idea found its development in works [16-20].

\section{Theoretical part}

Let $\mathrm{g}(\mathrm{r})$ be the distribution density of bubbles along the radius.

Let us assume that $\mathrm{g}(\mathrm{r})$ is the Gaussian distribution. It is determined by two parameters: the $R_{0}$ median size and the s standard deviation.

$$
g(r)=(s \sqrt{2 \pi})^{-1} \exp \left(-\left(r-R_{0}\right)^{2} / 2 s^{2}\right) .
$$

Let us assume that the $p(r)$ acoustic pressure created by a bubble is proportional to its size (the larger the bubble is, more noise it produces). Let's write it down as:

$$
p(r) \sim r .
$$

Given that we know the initial bubble size distribution:

$$
p(r) \sim r \cdot \sqrt{g(r)}==r \sqrt{(s \sqrt{2 \pi})^{-1} \exp \left(-\left(r-R_{0}\right)^{2} / 2 s^{2}\right)}
$$

Now let us pass to the frequency domain. To do this, the Minnaert relation will be used:

$$
f \approx M\left[m \cdot s^{-1}\right] / r .
$$

In regard to an air bubble in water:

$$
M \approx 3\left[m \cdot s^{-1}\right] .
$$

Let us rewrite expression 3 taking into consideration expression 4 :

$$
\frac{M}{f} \sqrt{(s \sqrt{2 \pi})^{-1} \exp \left(-\left(\frac{M}{f}-R_{0}\right)^{2} / 2 s^{2}\right)} .
$$

Note that $p(f)$ is the analog of bubbles noise spectrum. It is a mistake to assume that a peak in the spectrum would be observed at the frequency of $f_{0}=M / R_{0}$ corresponding to bubbles with the $R_{0}$ radius. In fact, the peak would be shifted to the left. Let us find this peak.

Certain simple calculations (taking the derivative and equating it to zero) will make it possible to find out that the maximum is reached at:

$$
f_{\max }=2 M\left(M / f_{0}+\sqrt{\left(M / f_{0}\right)^{2}+8 s^{2}}\right)^{-1} .
$$

Thus, if the bubbles noise spectrum is measured, the peak will be observed at the $f_{\max }$ frequency, which corresponds to bubbles with the following radius: 


$$
\begin{aligned}
& r_{\max }=M / f_{\max }= \\
& =\frac{1}{2}\left(M / f_{0}+\sqrt{\left(M / f_{0}\right)^{2}+8 s^{2}}\right)= \\
& =\frac{1}{2}\left(R_{0}+\sqrt{R_{0}^{2}+8 s^{2}}\right) .
\end{aligned}
$$

As could be seen from the obtained expression, the shifted size depends on the $s$ RMSE value. Now it is necessary to understand how to determine $f_{0}$ using the $f_{\max }$ from the measured spectrum.

Taking another look at expression 5, it becomes clear that the peak is shifting due to the $1 / f$ multiplier. It means, if we divide the measured spectrum graph by $(1 / f)$, then a curve with a maximum at the $f=f_{0}$ point is obtained:

$$
p(f) /(1 / f) \sim \sqrt{g(r)}
$$

Recalling that in the case of spectra it is easier to work in logarithmic quantities, let us logarithm the resulting expression:

$$
\begin{aligned}
& 20 \log (p(f) /(1 / f))= \\
& =20 \log (p(f))-20 \log (1 / f) .
\end{aligned}
$$

Thus. subtracting $20 \log (1 / f)$ from the measured spectrum displayed in $(\mathrm{dB})$ results in a curve with a maximum at the $f_{0}$ point. So, it became clear how to calculate the $R_{0}=M / f_{0}$ median bubble radius.

It remains only to determine the $s$ RMSE. To do this, $s$ should be expressed from expression 9 through rmax and $R_{0}$ :

$$
s=\sqrt{\frac{\left(2 r_{\max }-R_{0}\right)^{2}-R_{0}^{2}}{8}} .
$$

Thus, the $g(r)$ bubbles initial distribution with the $R_{0}$ median size and the $s$ standard deviation is found.

\section{Experimental part}

To confirm the theory, a number of experiments were carried out to determine the air bubbles size distribution in water. Experiments were performed in a cubic reservoir (1) with dimensions of $0.3 \times 0.3 \times 0.3 \mathrm{~m}$ (see Fig. 1).

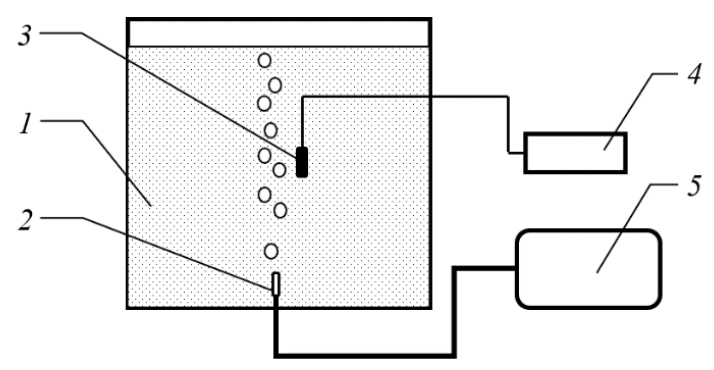

Fig. 1. Experimental installation diagram. 1 - cubic reservoir filled with water; $2-23 \mathrm{G}$ injection needle; 3 - Bruel \& Kjaer type 8103 hydrophone; 4 - Pulse LAN-XI spectrum analyzer; 5 - storage tank with air 
The reservoir was filled with water. The $23 \mathrm{G}$ injection needle (2) was used to supply air from the collection reservoir (5); and thus, bubbles were generated. Bubble size was controlled by the flow rate of supplied air. The higher the flow rate was, the larger were the bubbles.

Acoustic noise emitted by the bubbles was registered with a BK type 8103 hydrophone (3) connected to the Pulse LAN-XI spectrum analyzer (4). Then, registered signal was processed by the fast Fourier transform (FFT), and the noise spectrum was calculated. The hydrophone was positioned in the reservoir center next to the flow of bubbles.

In total, 4 experiments were conducted. The first experiment was carried out with the maximum flow rate of supplied air. In subsequent experiments, the air flow rate decreased.

\section{Results}

Results were processed as follows. From the measured spectrum of bubble noise, the $f_{\max }$ frequency corresponding to the peak was found. Further, the spectrum was corrected according to expression (12). Then, the $f_{0}$ frequency corresponding to the peak in the corrected spectrum was determined. After that, the $R_{0}$ and $r_{\max }$ bubble radii were calculated using the Minnaert relation (6). And finally, the $s$ RMSE was determined by formula (13). The $R_{0}$ and $r_{\max }$ found parameters were used to plot a graph of the air bubbles distribution according to the $g(r)$ size. Obtained distributions were compared with the distributions calculated from relation (11). Results are presented in Fig. 2.
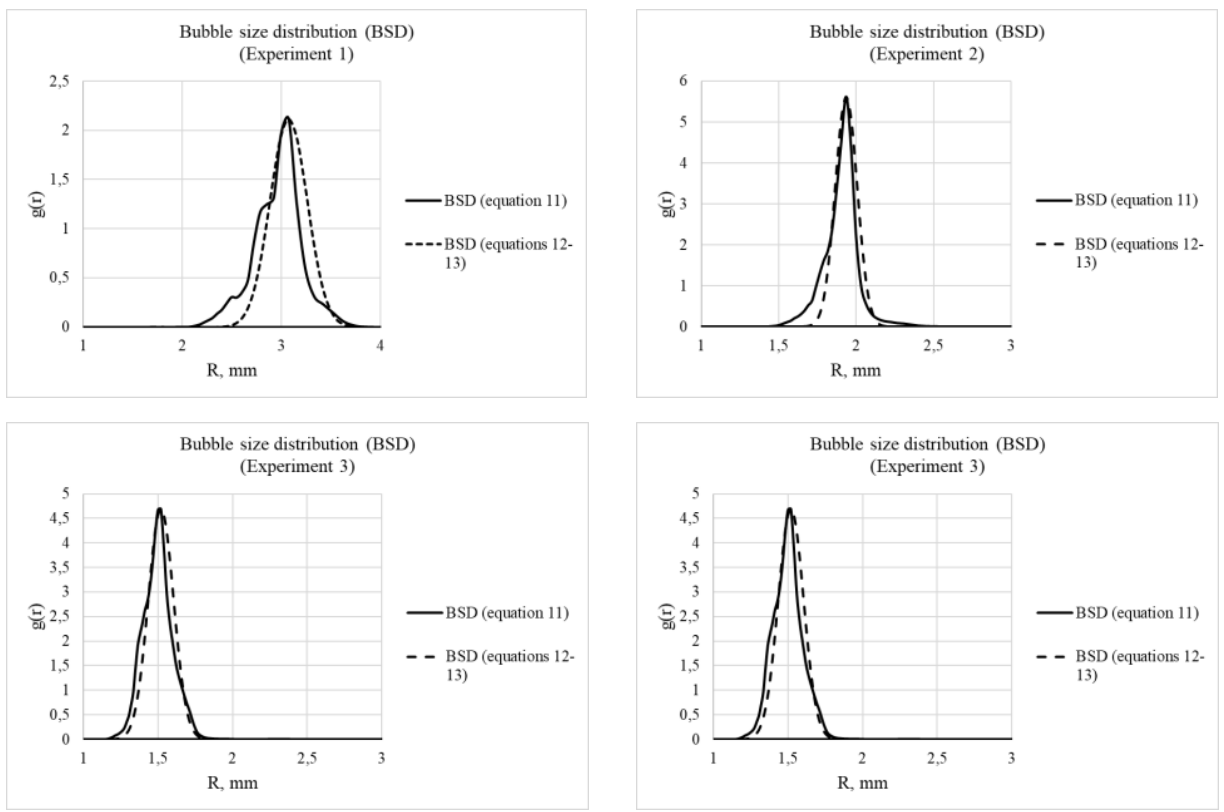

Fig. 2. Experimental results. Comparison of bubble size distributions according to size calculated in two different ways

As could be seen from the results of experiments, the bubble size depends on the flow rate of supplied air, the higher is the flow rate, the larger is the size. Distributions calculated from the corrected spectrum using relation (11) slightly differ from the Gaussian distribution. The larger are the bubbles, the higher is the deviation. This could be due to the fact that the acoustic wave frequency emitted by a bubble is inversely proportional to its size. 
Accordingly, with the decreasing frequency relative error in determining the $f_{\max }$ and $f_{0}$ frequency difference, which are used to calculate the Gaussian distribution parameters, increases. It should be noted that for bubbles with a radius less than $1.5 \mathrm{~mm}$ (experiments 4 and 5), distribution is almost identical to the Gaussian one.

\section{Conclusion}

This work proposes a passive acoustic method for air bubbles size determination in water. Advantage of this method is that, unlike active methods, it is invasive, i.e. it does not affect the gas-liquid flow being studied. Second part describes the mathematical model of recalculating the bubble noise spectrum into the bubble size distribution, and it was tested on the experimental installation under various operating modes with the median bubble radii ranging from 1.3 to $3 \mathrm{~mm}$.

\section{References}

1. A. A. Aleksandrov, V. A. Akat'ev, M. P. Tyurin, E. S. Borodina, and O. S. Kochetov, Her. Bauman M oscow State Tech. Univ. Ser. Nat. Sci. 46 (2018).

2. B. S. K senofontov and V. P. Y akushkin, in 29th Int. Miner. Process. Congr. IMPC 2018 (Canadian Institute of M ining, M etallurgy and Petroleum, Department of E cology and Environment Protection, B auman M oscow State Technical University, 2-Y a Baumanskaya, 5, M oscow, 105005, Russian Federation, 2019), pp. 1063-1074.

3. M. V Ivanov and B. S. K senofontov, W ater Sci. Technol. 69, 1434 (2014).

4. Y. Zhang and H. Sun, Proc. - Int. Conf. Commun. Syst. N etw. Technol. CSNT 2012 671 (2012).

5. T. Gaillard, C. H onorez, M. J umeau, F. Elias, and W. Drenckhan, Colloids Surfaces A Physicochem. Eng. A sp. 473, 68 (2015).

6. R. M azahernasab and R. A hmadi, Physicochem. Probl. M iner. Process. 52, 690 (2016).

7. T. F. A rgo, P. S. Wilson, and V. Palan, J. A coust. Soc. A m. 123, EL 121 (2008).

8. N. F. Bunkin and F. V B unkin, Physics-U spekhi 59, 846 (2016).

9. V. A. Babenko, N. F. Bunkin, and A. A. Sychev, Quantum Electron. 47, 901 (2017).

10. X. J. Wu and G. L. Chahine, J. Hydrodyn. 22, 325 (2010).

11. M. Christensen and P. Thomassen, 121 (2014).

12. J. Xue, 1 (2004).

13. W. A . Al-M asry, E. M. Ali, and Y. M. A qeel, Chem. Eng. Res. Des. 83, 1196 (2005).

14. P. K. V olkov, Gas-fluid mechanics 75 (1996)

15. T. Leighton, (1994).

16. J. W .R. Boyd and J. V arley, Chem. Eng. Sci. 56, 1749 (2001).

17. R. M anasseh, R. F. L aFontaine, J. Davy, I. Shepherd, and Y .-G. Zhu, Exp. Fluids 30, 672 (2001).

18. C. A. Greene and P. S. Wilson, J. A coust. Soc. A m. 131, EL 61 (2012).

19. S. Husin and D. M ba, II, 0 (2010).

20. L. Chen, S. W ood, S. M oore, and B. N guyen, Proc. A coust. 1 (2012). 